\title{
Antidepressant Effects Associated With Different Exercise Conditions in Participants With Depression: A Pilot Study
}

\author{
Fabien Legrand ${ }^{1}$ and Jean Philippe Heuze ${ }^{2}$ \\ University of Reims and ${ }^{2}$ Joseph Fourier University
}

\begin{abstract}
In this article, we examined the antidepressant influence of an 8-week-long aerobic exercise intervention in which two training parameters were manipulated: exercise frequency and group environment. Twenty-three individuals with elevated symptoms of depression were recruited in a sport and fitness facility and agreed to participate in this 8-week study. They were randomly assigned to three groups: (a) low-frequency exercise (control) $(n=7)$, (b) high-frequency exercise $(n=8)$, and (c) high-frequency exercise + group-based intervention $(n=8)$. Participants completed the Beck Depression Inventory (BDI-II) at study entry, and at 4 and 8 weeks subsequently. The results showed that those in the high-frequency aerobic exercise experimental groups reported lower depression scores than those in the low-frequency (control) group at 4 weeks $(13.2 \pm 7.3$ and $11.7 \pm 3.1$ vs. $22.4 \pm$ $7.5)$ and 8 weeks $(10.9 \pm 8.1$ and $9.6 \pm 2.5$ vs. $20.7 \pm 6.3)$. However, alleviation in depressive symptoms was not found to be greater in those participants who received a group-based intervention.
\end{abstract}

Key Words: aerobic exercise, depression, exercise conditions

Major symptoms of depression include a lowered mood or loss of interest and pleasure, loss of appetite, weight loss/gain, sleep disturbance, psychomotor agitation or retardation, energy decrease, sense of worthlessness, guilt, difficulty in concentrating, or thoughts of suicide (APA, DSM-IV-TR, 2000). These symptoms can be referred to as clinically defined depression if formal diagnosis has been established through reliable and commonly used assessment procedures in psychiatry, such as the Research Diagnostic Criteria (RDC; Spitzer, Endicott, \& Robins, 1978). However, several validated self-report instruments as well as normal ranges for their item scores have been developed to measure the level of depressive symptoms (e.g., Self-Rating Depression Scale, Zung, 1965; Beck Depression Inventory, Beck, Steer, \& Brown, 1996) so that it makes it possible to use these instruments to identify cases or patients. For example, scores above 16 on the Beck Depression Inventory have often been viewed as highly suggestive of clinical depression in many exercise-based intervention studies (Mutrie, 2000). 
The prevalence of lifetime depression is between $13 \%$ and $17 \%$ in most developed countries (e.g., United States: 16.2\% according to Kessler and colleagues, 2003; France: $14.9 \%$ in the survey by Lepape, 1998). It is now acknowledged that depression has high personal, social, and economic costs. For example, Greenberg and colleagues (2003) noted that in 2000, the economic burden of depression was $\$ 83.1$ billion in the United States, including direct medical costs ( $\$ 26.1$ billion), suicide-related mortality costs ( $\$ 5.4$ billion), and workplace costs ( $\$ 51.5$ billion).

Most cases of depression are treated in general practice (antidepressant drugs are the most frequently delivered treatment), with a much lesser proportion (the most severe cases) referred to psychiatric services (e.g., Schulberg, Katon, Simon, $\&$ Rush, 1999). However, research has illuminated the adjunctive value of exercise in the treatment of depression (for review, see the meta-analysis by Craft and Landers, 1998). Indeed, the literature in this area, which has been growing over the past 30 years, supports the hypothesis that exercise can have therapeutic benefits for people diagnosed with mild-to-moderate clinical depression. As an illustration, the recent studies by Dunn and colleagues (Dunn, Trivedi, Kampert, Clark, \& Chambliss, 2002, 2005) highlighted that a 12-week long program of aerobic exercise at a dose of $17.5 \mathrm{kcal} \cdot \mathrm{kg}^{-1} \cdot \mathrm{week}^{-1}$ stands as an effective treatment for major depressive disorder of mild-to-moderate severity (whereas aerobic exercise at a dose of 7.0 $\mathrm{kcal} \cdot \mathrm{kg}^{-1} \cdot \mathrm{week}^{-1}$ yielded antidepressant effects comparable to that of a placebo treatment). In addition, nonaerobic forms of exercise (e.g., strength and flexibility exercises) have appeared to provide participants with reductions in depression similar to those reported in aerobic exercise programs (Martinsen, Hoffart, \& Solberg, 1989). The antidepressant effects of exercise have usually been found to be large and comparable to those for traditional therapies (e.g., Blumenthal et al., 1999; Klein et al., 1985). For example, though patients with clinical depression receiving antidepressant drugs exhibited the fastest initial response, participants who exercised experienced similar reductions on BDI scores after 16 weeks of treatment (Blumenthal et al., 1999).

However, much research in this area has been characterized by a number of methodological shortcomings (e.g., inappropriate population, lack of control groups or random assignment of participants, experimenter expectancy effects) and practice inconsistencies regarding type of physical activities, program duration, number of sessions per week, and relative intensity. As a matter of fact, in spite of previous meta-analysis by Craft and Landers (1998) or North, McCullagh, and Tran (1990)_all suggesting that chronic exercise is effective in alleviating symptoms of depression-Lawlor and Hopker (2001) more cautiously concluded that

"it is not possible to determine from the available evidence the effectiveness of exercise in the management of depression. . . However, exercise may be efficacious in reducing the symptoms of depression in some volunteers in the short term . . . but this should not replace standard treatment, particularly for those with severe disease." (p. 7)

There is a need to carry out methodologically sound empirical studies to compare the psychological response resulting from varying exercise conditions in individuals with clinical depression. To date, little is known about these issues because only minimal attention has been devoted to exercise training parameters (i.e., duration, frequency, and intensity). It may be that exercise prescription for the purpose of 
depression treatment differs from the amount needed for general health or fitness benefits. For example, high-intensity exercise (i.e., physical activity requiring up to $90 \%$ of one's age-adjusted maximal heart rate) is prescribed for optimal physical conditioning (ACSM, 2000) but has been associated with inconsistent changes in mood, and sometimes, with undesirable changes (Motl, Berger, \& Davis, 1997).

With regard to the aforementioned considerations, we proposed first that (a) regulating day-to-day variability in mood for people with elevated symptoms of depression may be an effective way to relieve their depressive feelings and (b) frequency is a key point for designing exercise programs suited to reestablishing positive mood in participants with elevated symptoms of depression. Indeed, although the exact time frame for the psychological response to exercise is not clear, mood changes seem to dissipate within $4 \mathrm{hr}$ of ending an exercise session (Thayer, 1996; Petruzzello \& Landers, 1994). As a result, if reestablishing and maintaining an enhanced mood in persons with depression is the major goal, there is a need to exercise on a frequent basis. Therefore, in the present study, our first hypothesis was that frequent exercise would lead to more favorable changes in depressive symptoms as measured by the Beck Depression Inventory (BDI-II) than infrequent exercise. Findings from the randomized controlled trial by Dunn and colleagues (2005) did not support this contention, but it is our opinion that they actually compared two high-frequency exercise conditions (i.e., three sessions/week vs. five sessions/week). Indeed, in the United States, $40-45 \%$ of the general population are sedentary, and, among active Americans, most report having less than three weekly sessions of physical activity (USDHHS, 2000). For our purpose, low-frequency exercise was defined as an exercise regime of one weekly session, and high-frequency exercise consisted of three to five exercise bouts per week. An important concern of ours related to some of the common symptoms of depression (i.e., fatigue, lack of energy, psychomotor retardation, hopelessness, and feelings of worthlessness) that may interfere with the motivation to exercise (especially for the high-frequency exercise condition, which may be perceived as too demanding). With regard to this issue, it was decided to provide participants from both groups with very close supervision of the exercise sessions and face-to-face intervention.

A secondary aim of this pilot study was to examine the process of mental health change through physical activity. Indeed, Faulkner and Carless (2006) recently underlined that understanding how exercise affects depression was critical in order to provide the most effective physical activity intervention and to decrease the risk of missing some of the most powerful psychosocial effects. The mental health literature points out several plausible mechanisms operating within this process (see Fox, 1999, for a review) and, among them, acknowledges the central importance of interrelations with others in achieving mental health (e.g., Faulkner \& Carless, 2006). Indeed, the literature has provided empirical evidence for a negative relationship between depression and social relations (Henderson, 1998). Depression is associated with less support, few social ties, and more problems in social relationships. Moreover, social relations have been posited to mediate the effects of physical activity on depression (Bailey \& McLaren, 2005; Deforche \& DeBourdeaudhuij, 2000). Because many physical activities are performed with others (Coleman \& Iso-Ahola, 1993), collective exercise sessions may be thought to provide a sense of social integration (Andersson, 1998) and an opportunity to expand participants' social networks (Stathi, Fox, \& McKenna, 2002). Therefore, 
this pilot study also investigated the possible impact of social relations created within an exercise group on depression.

However, previous studies on the role of social relations in the physical activity-depression relationship have produced equivocal findings (Bailey \& McLaren, 2005; O'Neal, Dunn, \& Martinsen, 2000), particularly because simply performing activities with others is not always associated with an increase in social relationships (Bailey \& McLaren, 2005). So, in exercise settings, it has now become a common practice to foster social interaction by implementing team-building interventions based on group or class cohesion studies from the social psychology literature (e.g., Carron \& Spink, 1993; Estabrooks \& Carron, 1999).

Cohesion refers to a social process that keeps members of a small group together and united to varying degrees. In sport and exercise settings, cohesion is generally defined as "a dynamic process that is reflected in the tendency for a group to stick together and remain united in the pursuit of its instrumental objectives and/or for the satisfaction of members' affective needs" (Carron, Brawley, \& Widmeyer, 1998; p. 213). Thus, this construct refers to the bonding within a group that links members together and has been found to be negatively related with depression and tension (Terry et al., 2000), but positively related with satisfaction (Widmeyer \& Williams, 1991), communication between participants (Hill \& Estabrooks, 2000), and positive affective states (Courneya, 1995). In light of all these studies, we have good reasons for thinking that class cohesion might also help depressed participants to reestablish a positive mood. As a second hypothesis, then, we suggested that antidepressant effects associated with high-frequency training would be enhanced with physical activities that are performed within a cohesive group.

In summary, the following hypotheses were tested:

1. High-frequency exercise training will result in greater decreases in depressive symptoms than infrequent exercise.

2. Those participants in the high-frequency exercise training condition who are receiving a group intervention aiming at enhancing one's perception of cohesiveness will report the most significant decreases in depression.

\section{Method}

\section{Participants}

The study was performed in a commercial sport and fitness center ("YNS") in Reims, France. All participants were regular customers of this sport and fitness facility and were recruited by the first author over a 3-month period by direct onsite advertisement and on YNS Web page. There were 107 potential participants at the end of this period. They were considered for study participation if (a) they were aged 18 and over; (b) they had a BDI score of 16 or above; (c) they did not receive any treatment for depression; (d) they exercised fewer than three times per week and $20 \mathrm{~min}$ for each bout; and (e) they were able to read, understand, and provide written responses in French language.

Twenty-nine individuals $(27.1 \%)$ met all five conditions, but only twenty-five accepted to take part in this study and were then randomly allocated to one of three groups: low-frequency exercise (control group), high-frequency exercise (HFex), 
and high-frequency exercise + group-based intervention $(\mathrm{HFex}+\mathrm{GBI})$ (see Table 1). Eligibility was ensured by a screening face-to-face interview that occurred 2 or 3 days before the beginning of the program. The BDI was administered again, and biographic information regarding length of attendance and current exercise regime was gathered (see Table 1). Participants were also informed that (a) the study was 8 weeks in length and consisted of "an individualized training program aiming for the improvement of one's physical fitness and quality of life," (b) two periods of assessment were planned (after 4 weeks and at the end of the program), and (c) the first author would be present at the fitness center Monday through Friday during designated hours (8-11 am, 1-3 pm, and 7-9 pm) allocated to the research project for exercise supervision so as to provide encouragement and maximize participation. Finally, informed consent was obtained from each participant, mentioning that he/she should feel free to discontinue his/her involvement in the study at any point, and that this experiment involved a minimal overall attendance of 7 exercise bouts (i.e., $87 \%$ of the 8 planned sessions throughout our 8-week-long intervention; see "Procedure and Design" section) for the low-frequency group and 21 exercise bouts (i.e., $87 \%$ of the 24 planned sessions for a three-sessions-per-week participation throughout our 8-week intervention; see "Procedure and Design" section) for the high-frequency groups. Unfortunately, two men (one in the low-frequency (control) group and one in the high frequency + group-based intervention group) subsequently failed to meet these minimal requirements and were accordingly excluded from analysis. As a result, our final sample included only 23 participants, whereas an estimated sample size of 28 individuals was needed to demonstrate a statistically significant difference in depression reduction (defined as a reduction of 1 standard deviation or above) between treatments at a $p$ value from .05 and a power of $70 \%$. This discrepancy between needed and actual sample size was an important concern and is further addressed in the discussion section.

Table 1 Descriptive Statistics at Baseline $(n=23)$

\begin{tabular}{lccc}
\hline & $\begin{array}{c}\text { HFex } \\
(\boldsymbol{n}=8)\end{array}$ & $\begin{array}{c}\text { HFex+GBI } \\
(\boldsymbol{n}=8)\end{array}$ & $\begin{array}{c}\text { Control } \\
(\boldsymbol{n}=7)\end{array}$ \\
\hline $\begin{array}{l}\text { Age (years) } \\
\quad M(S D)\end{array}$ & $31.13(8.17)$ & $38.00(12.61)$ & $34.43(11.13)$ \\
$\begin{array}{l}\text { Gender } \\
\quad \text { Female }(\%)\end{array}$ & $5(62.5)$ & $6(75.0)$ & $5(71.4)$ \\
$\quad \begin{array}{l}\text { Male }(\%) \\
\text { Current exercise regime (bouts/week) }\end{array}$ & $3(37.5)$ & $2(25.0)$ & $2(28.6)$ \\
$\quad \begin{array}{l}M(S D) \\
\text { How long have you been attending this facility? (years) }\end{array}$ & & & \\
$\quad \begin{array}{l}M(S D) \\
\text { Current depressive symptoms severity }\end{array}$ & $0.97(0.42)$ & $1.06(0.54)$ & $.89(0.61)^{*}$ \\
$\quad M(S D)$ & & & \\
\hline
\end{tabular}

Note. . Control = participants who were assigned to the low-frequency exercise condition; HFex = participants who were assigned to the high-frequency exercise condition; $\mathrm{HFex}+\mathrm{GBI}=$ participants who were assigned to the highfrequency exercise + group-based intervention condition

$* p>.05$. 


\section{Procedure and Design}

This pilot study was conducted from December 2004 to April 2005. The intervention program lasted 8 weeks, from the beginning of February to the end of March 2005. Although the meta-analysis by North and colleagues (1990) suggested that longer programs had greater antidepressant effects (effect size of -2.93 for 21-24 weeks, but effect size of only -0.11 for less than 4 weeks), a recent study found that 10 days of moderate walking triggered a clinically relevant reduction in depression scores for patients with a major depressive episode (Dimeo, Bauer, Varahram, Proest, \& Halter, 2001). Moreover, the vast majority of people with depression who receive psychotherapy (a standard form of antidepressant therapy) attend fewer than 10 sessions, and the number of sessions required to achieve a 50\% patient improvement rate has appeared to range between 5 to 18 sessions (e.g., Hansen, Lambert, $\&$ Forman, 2002). Finally, most antidepressant drugs have also been found to take effect in a short time, with an onset of therapeutic action usually coming around 3 weeks (e.g., Angst \& Stassen, 2001).

Given the purpose of the present pilot study (examining a high-frequency exercise training condition and preventing the participants with depression from dropping out because of the program prescribed being too demanding), a short duration of 8 weeks was felt to be appropriate. This time period also seems to be consistent with the development of cohesion (e.g., 6 weeks in the second study by Estabrooks \& Carron, 1999).

As mentioned above, participants were randomly assigned in a 3 (Exercise training condition: low-frequency exercise, high-frequency exercise [HFex], high frequency exercise + group-based intervention $[\mathrm{HFex}+\mathrm{GBI}]) \times 3$ (Time of depression assessment: baseline, Week 4, Week 8) mixed-model design with repeated measures on the last factor.

In line with suggestions by Berger and Motl (2001), the training sessions that were proposed (a) promoted rhythmical abdominal breathing (i.e., aerobic activities performed at one's target heart rate (THR), which was $60-80 \%$ of one's maximal heart rate), (b) were noncompetitive, (c) did not require the participants to be vigilant for constantly changing environments, and (d) involved rhythmical and repetitive movements. The rationale behind these requirements is based on previous research evidence indicating that certain practice conditions can maximize the psychological benefits of exercise, including mood enhancement (e.g., Berger \& Owen, 1988).

Participants in the low -frequency group $(n=7)$ had to report to the sport and fitness center as often as they used to do before the beginning of the study. Their usual training activities were not predominantly aerobic (i.e., bodybuilding exercises, stretch classes), so they were asked to perform one aerobic activity (e.g., brisk walking, running, biking on a stationary bicycle) for a duration of approximately $30 \mathrm{~min}$ per week within their THR. Relative intensity was controlled by a pacemaker wristwatch with a heart rate monitor chest band (Polar Heart Rate Monitor). Along with the watch readout, the heart rate monitor also had an alarm that sounded when heart rate became too high ( $>80 \%$ of one's age-adjusted maximal heart rate) or too low ( $<60 \%$ of one's age-adjusted maximal heart rate), allowing participants to adjust their effort accordingly by themselves. Recent recommendations have argued that subjects who are at least minimally physically active should compose the "control" group in exercise studies (Booth \& Lees, 2006). 
The rationale for this recommendation is that physical inactivity and chronic disease are inevitably linked, so that it makes it more likely to find improved health outcomes in physically active groups when compared with sedentary groups, whatever the treatment. So, in this report, the low-frequency exercise group was referred to as the "control" group.

In the second condition (i.e., high-frequency aerobic exercise training [HFex]; $n=8$ ), exercise frequency was set at 3-5 sessions per week. This exercise frequency was felt to represent a significant increase in participants' regular exercise schedule, which typically included only one weekly session (Table 1). The other training requirements (i.e., intensity and duration) were similar to those used in the first condition (see above). Participants had to complete their scheduled session individually on a motorized treadmill, a stationary bicycle, or a rowing ergometer under the first author's supervision. It should be emphasized that exercise sessions were not scheduled at the same time for all the participants since (a) some had a job that prevented them from coming to the fitness facility during office hours and (b) exercising together (toward collective goals and/or for social purposes) would most certainly have enhanced one's perception of group cohesiveness.

Finally, the last condition (i.e., high-frequency aerobic exercise training + group-based intervention $[\mathrm{HFex}+\mathrm{GBI}] ; n=8$ ) involved exercise training requirements identical to those of the second group (see above) but was structured so as to develop participants' perception of group cohesiveness. Our team-building intervention followed the guidelines provided in previous studies (i.e., group distinctiveness, group goals, and interaction and communication between group members; Carron \& Spink, 1993; Estabrooks \& Carron, 1999). The strategies implemented included the following:

1. Having collective training sessions for the total duration of the study

2. Encouraging participants to chat and to cheer each other on

3. Asking participants to wear group T-shirts with the sport and fitness center logo

4. Assigning the participants in this group the goal of reaching a 50-mile mark on the center's treadmills by the end of the study (they were informed that this goal would be attained by having three or four members perform a 1.5-mile walk/run at each exercise session).

\section{Participants' Safety}

Although no specific safety measure was phased in, we had available some names and phone numbers of local resources so that any participant with significant emotional or psychological problems during an exercise session could possibly get in touch with health care providers.

\section{Outcome Measures}

Depression. The primary outcome measure was the change in the Beck Depression Inventory (BDI-II; Beck et al., 1996) score from baseline to 8 weeks. The BDI-II was selected because it measures severity of symptoms of clinical depression and is widely used in efficacy studies of antidepressant treatments. It is a 21- 
item self-report questionnaire in which each item consists of several statements describing symptoms of depression. Respondents are asked to circle the statement that best describes their symptoms related to that particular aspect of depression (i.e., feeling of guilt, changes in sleep). The statements are numbered from 0 to 3 , with higher numbers indicating more severe symptoms. Scores for responses are summed to obtain an overall score. In the present study, each participant completed the BDI-II individually in a private room, at study entry, 4 weeks, and 8 weeks. Internal consistency estimates observed in the present investigation, respectively, at Time 0 , Time 1, and Time 2, were $\alpha=.76, .95$, and .88 .

Beck and colleagues suggest the following cut score guidelines: scores of 0-13 indicate "minimal" depression, 14-19 indicate "mild" depression, 20-28 indicate "moderate" depression, and 29-63 indicate "severe" depression. So remission in depressive symptoms can be defined as a BDI-II score of $\leq 13$.

Social Manipulation Check. Reactions to the group intervention were measured by plotting changes in participants' scores of perceived cohesion. In the sport and exercise literature, cohesion is generally considered from the perspective of a conceptual framework advanced by Carron and his colleagues (Carron et al., 1998; Carron \& Brawley, 2000), and composed of four dimensions: (a) Individual Attractions to the Group-Task (ATG-T), the individual member's perceptions of his or her personal involvement with the group task; (b) Individual Attractions to the Group-Social (ATG-S), the individual member's perceptions of his or her personal acceptance and social interaction with the group; (c) Group Integration-Task (GI-T), the individual member's perceptions of the similarity, closeness, and bonding that exists within the group as a totality around its collective task; (d) Group IntegrationSocial (GI-S), the individual member's perceptions of the similarity, closeness, and bonding that exists within the group as a totality around social concerns.

Given that the secondary aim of this study focused on social relations, only the social components of cohesion were considered (ATG-S [5 items] and GI-S [4 items]) and assessed through the Questionnaire sur l'Ambiance du Groupe (QAG; Heuzé \& Fontayne, 2002). Following the protocol advanced by Carron and Brawley (2000), minor changes to the wording of QAG items were made to better reflect the exercise context. Each item is scored on a 9-point scale anchored at the extremes by strongly disagree (1) and strongly agree (9). Participants' responses on each scale were averaged to produce a scale score, with larger scores reflecting stronger perceptions of cohesiveness. The validity and reliability of the QAG have been attested in the studies undertaken to develop it (Heuzé \& Fontayne, 2002; Studies 4 and 5).

As mentioned above, the QAG was used in order to control for the efficiency of the team-building intervention. In this study, therefore, only the participants of the high-frequency aerobic exercise training with group-based intervention condition completed it twice, at Week 4 and Week 8. This specific timing ensured that team members would have sufficient opportunities to interact and develop group beliefs before the first assessment of cohesion. The timing of the first assessment was also congruent with previous research conducted in exercise setting (e.g., Loughead \& Carron, 2004; Loughead, Colman, \& Carron, 2001). Cronbach alpha values computed with the sample used in the present study, respectively at Time 1 and Time 2, were as follows: ATG-S, $\alpha=.67$ and .71; GI-S, $\alpha=.75$ and .79 . 


\section{Data Analysis}

This study employed a mixed between-within subject design in which there are two independent variables, (a) one between-subject variable (i.e., exercise training program with three conditions), and (b) one within-subject variable (i.e., testing time with three occasions of assessment). The dependent variable is depression as measured by the BDI-II. As a result, we performed a 3 (Groups) $\times 3$ (Time points) analysis of variance with repeated measures on the last factor. The significance level was $\alpha=.05$.

Before analysis, depression scores were examined through various SPSS programs in order to test for the normality of sampling distributions (ANOVA assumes that the sampling distribution of means are normal within each group) as well as the homogeneity of variance within each cell of the design. There were no univariate within-cell outliers at $\alpha=.05$, and results regarding assumptions of distributions normality and homogeneity of variances were satisfactory. A one-way ANOVA was further conducted to check for preexisting group differences on BDI-II scores at baseline, the result of which yielded no significant difference.

\section{Results}

Participants in both groups completed the program without interruption, and no training related complications were observed during the study. To examine whether the team-building intervention resulted in a development of cohesion, one-way withinsubjects ANOVAs were performed for each dimension of the QAG. Results revealed a significant difference between Time 1 and Time 2 for GI-S, $F(1,7)=12.12, p=$ $.01, \eta^{2}=0.67$, power $=0.83$, indicating an increase in GI-S from Time $1(M=5.78)$ to Time $2(M=6.61)$.

Depression scores across time are shown in Figure 1 for each experimental group. To test our first hypothesis that different degrees of antidepressant effects would result from our three experimental conditions, a Group (control, high-frequency aerobic exercise training, high-frequency aerobic exercise training + group-based intervention) $\times$ Time (baseline, Week 4, Week 8) repeated measure ANOVA was conducted. The Group $\times$ Time interaction was significant, $F(4,40)=7.99, p<.01$, and follow-up pairwise multiple comparisons (Tukey's HSD test) indicated that the exercise treatment groups were less depressed than the control group at 4 and 8 weeks.

Nevertheless, contrary to our second hypothesis, there was no statistical evidence supporting a greater decrease in depression scores among participants who received the group-based intervention (Figure 1).

Scores at last observation (Table 2) revealed that at the end of our study, 13 participants out of those 16 including the high-frequency aerobic exercise groups had depression scores indicating no evidence of clinical depression (i.e., BDI score $\leq 13$ ).

Both high-frequency exercise groups had a very important reduction in depression from baseline to Week 4 -for the HFex condition, score change $=7.13, t(7)=$ 7.92, $p<.01$, and for the HFex+GBI condition, score change $=7.63, t(7)=6.16, p$ $<.01$. The high-frequency exercise groups also had a much more attenuated, albeit still statistically significant, reduction from Week 4 to Week 8 -score change in the HFex condition $=2.37, t(7)=2.37, p=.05$, and score change in the HFex+GBI condition $=2.12, t(7)=2.57, p<.05$. 


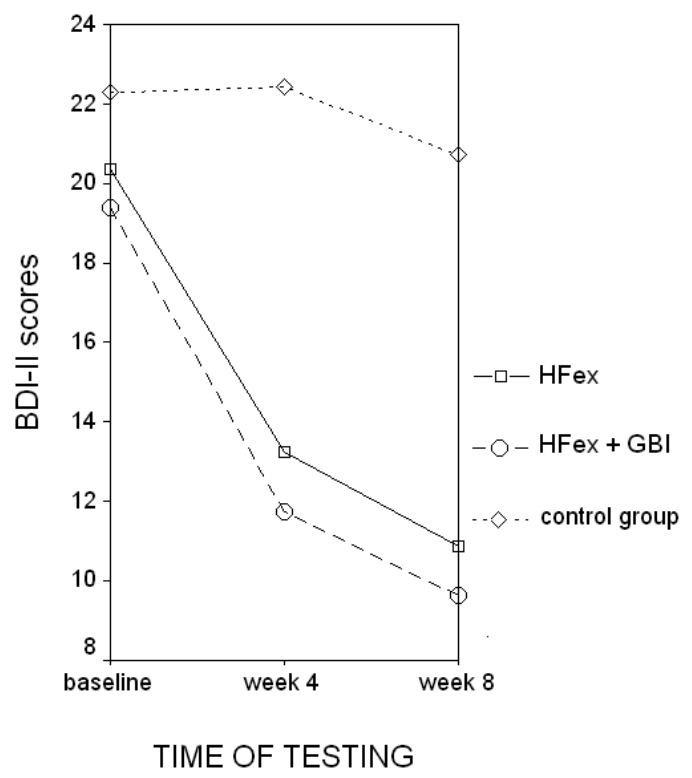

Figure 1 - Group $\times$ Time interaction for BDI-II scores.

Table 2 Scores at Last Observation

\begin{tabular}{lcccc}
\hline Group & $n$ & BDI Mean $(S D)^{\mathrm{a}}$ & Response (\%) $^{\mathrm{b}}$ & Remission (\%) $^{\mathrm{c}}$ \\
\hline Control & 7 & $20.71(6.26)$ & $0(0 \%)$ & $1(14.3 \%)$ \\
HFex & 8 & $10.88(8.08)^{*}$ & $5(62.5 \%)$ & $6(75 \%)$ \\
HFex+GBI & 8 & $9.63(2.5)^{*}$ & $5(62.5 \%)$ & $7(87.5 \%)$ \\
Total & 23 & $13.39(7.6)^{*}$ & $10(43.5 \%)$ & $14(60.9 \%)$ \\
\hline
\end{tabular}

Note . BDI $=$ Beck Depression Inventory; control $=$ participants in the low-frequency exercise condition; $\mathrm{HFex}=$ participants in the high-frequency exercise condition; $\mathrm{HFex}+\mathrm{GBI}=$ participants in the high-frequency exercise + group-based intervention condition.

${ }^{a}$ For the sample taken as a whole, mean (SD) BDI-II score at baseline was 20.7 (5.08).

${ }^{b}$ Percentage with BDI-II $\leq 50 \%$ of participant's score at baseline.

'Percentage with BDI-II $\leq 13$.

$* p \leq .05$ vs. control.

\section{Discussion}

We first hypothesized that both high-frequency aerobic exercise stimuli (but not the low-frequency exercise group) would trigger a significant decrease in depression. This was supported by the data. Indeed, at study entry, all participants had "moderate" levels of clinically defined depression based on their scores on the BDI-II. At the conclusion of the study, however, only those from the control group still were moderately depressed (their mean BDI score at the end of the program was around 21 , and only one participant had a BDI score $\leq 13$ ), whereas most participants in the 
high-frequency exercise groups were not depressed anymore (mean BDI score at the end of the program was around 10 for the two high-frequency exercise groups and 13 participants out of 16 had a BDI score $\leq 13$ ). The mean difference in BDI scores between high-frequency exercise groups and the low-frequency exercise group at 8 weeks (i.e., scores at last observation) was -10.46 (95\% CI: -16.06 to -4.85). This difference appears to exceed the values usually found in previously published studies comparing exercise and no-treatment groups of participants and that used the BDI as a measure of depression (e.g., in their meta-analysis, Lawlor and Hopker (2001) came to a weighted mean difference of -7.3). However, some previous similar reports with short-term programs of aerobic exercise (i.e., $<10$ weeks) also showed differences of this magnitude or greater (e.g., Martinsen, Medhus, \& Sandvik, 1985; Mutrie, 1988). For example, in her 1988 study, Mutrie found a mean difference in BDI scores of -11.9 between aerobic exercise (three sessions of 20 min per week for 4 weeks) and control groups (95\% CI: -16.7 to -7.1 ).

Because participants in the control group exercised once a week, it should be concluded that moderate/vigorous aerobic training may have an antidepressant effect in people with moderate clinical depression provided that they exercise on a frequent basis (three to five weekly sessions). This exercise regime is consistent with current training guidelines for health-related fitness. In the United States for example, the joint recommendation of the Centers for Disease Control and the American College of Sports Medicine is that "every U.S. adult should accumulate 30 min or more of moderate-intensity physical activity on most, preferably all, days of the week." (Pate et al., 1995, p. 402).

Several reasons may account for the fact that high-frequency exercise is more likely to be associated with psychological benefits in individuals with depression. First, frequent and regular exercise presumably increases the fitness levels of participants, so, as the conditioning process develops, physical discomfort decreases and the exercise session becomes more enjoyable. Actually, several significant metabolic and physiological adaptations to aerobic exercise have been found to occur quite quickly (by the end of the first week of training), especially in participants with low fitness levels (like most depressed individuals) (Hickson, Hagberg, Ehsani, \& Holloszy, 1981). This should be considered as a reasonable hypothesis because, in our study, depressive symptoms were found to be substantially lowered after only 4 weeks (by the midpoint of the treatment). It is important to note that the delay for this exercise-induced antidepressant effect is particularly brief and, as we mentioned above, very close to the time course of any antidepressant drug (e.g., Angst \& Stassen, 2001). This finding that aerobic exercise programs of short duration can produce substantial improvement in participants with elevated symptoms of depression has already been highlighted in recently published studies (e.g., Dimeo et al., 2001).

Another way frequent exercise reduces physical discomfort is known as the selflearning process. Namely, with frequent participation, exercisers learn to interpret physical sensations associated with energy expenditure: they grow accustomed to deep breathing, sweating, and may even become adept at relaxing while exercising. As a result, sensations of discomfort and displeasure are less likely to impede participants' enjoyment.

Third, as suggested previously, even if little is actually known regarding the dynamics of affective response following exercise, some studies indicated that the 
exercise-induced psychological benefits usually last for 2 to 4 hours (e.g., Thayer, 1996). This time frame highlights the need for frequent participation in order to reestablish enhanced mood states. At this point, only speculative explanations can be proposed to reinforce this view. For example, as shown recently, it is likely that involvement in an exercise program is associated with a decreased tendency to use rumination in clinically depressed participants as well as enhanced perceptions of self-efficacy (Craft, 2005). Rumination refers to one's propensity to focus on one's negative feelings, thus leading to negative attributions about the self, past, present, and future, which can contribute to a continued depressed mood (Lyubomirski \& Noelen-Hoeksema, 1995). So, the more the depressed individual is busy with an engaging activity (like physical activity), the more he or she focuses on something other than his or her depressed mood.

Finally, frequent exercise might have antidepressant properties because of its repeated effects on the neurochemistry of mood regulation. Antidepressant medications usually exert an antidepressant effect by increasing the availability of neurotransmitters at receptor sites. The central serotonergic systems in particular are the targets for the selective serotonin reuptake inhibitors (SSRIs-widely used antidepressant drugs), which are believed to influence the metabolism and availability of serotonin. Previous investigations by Chaouloff (1997) showing that acute exercise increases brain serotonin are strongly evocative of a parallelism between the mechanisms underlying the antidepressant effects of exercise and those involved in the therapeutic properties of SSRIs. Once again, it should be mentioned that our results are of clinical relevance because the observed outcomes revealed a substantial antidepressant effect of high-frequency aerobic exercise by the fourth week of the study, namely, a time frame that is very close to the latency times of 2 to 4 weeks usually required for any therapeutic effect to occur with SSRIs (e.g., Einarson, Iskedjian, Addis, \& Mittmann, 1998). Several other lines of research using animal models of stress and depression have suggested that exercise may also decrease depression because it increases levels of brain norepinephrine (e.g., Dishman, Renner, White-Welkley, Burke, \& Bunnell, 2000) and/or brain-derived neurotrophic factors (BDNF; e.g., Cotman \& Engesser-Cesar, 2002).

With regard to the second hypothesis, to directly assess the additive effects of a group-based intervention on exercise-induced psychological relief, no reliable significant differences were found. Specifically, predictions that additional increases in the reduction of depressive symptoms resulting from our group-based intervention would be obtained among participants exercising frequently were not confirmed, with participants from both high-frequency exercise groups reporting similar amounts of reduction in depressive symptoms across time.

Although these results did not support our hypothesis, they make a valuable contribution to the health literature. Indeed, these findings suggest that social relations within an exercise group do not play an important part in the relationship between physical activity and depression over a short period of time (i.e., 8 weeks in this study).

This comes in contrast with Courneya's study (1995), which showed that higher perceptions of cohesion were associated with more positive affect during exercise. Nevertheless, this previous research was correlational in nature so that we cannot be sure whether increased perceptions of cohesiveness resulted in more positive affect or the other way round. Moreover, the participants were not 
depressed individuals, making it difficult to compare Courneya's results and those found in the present study.

One possible explanation for why our group-based intervention did not lead to further psychological benefits relates to the quality of social relations. Indeed, the association between depression and social relations is strongest for relations involving intimate members of the depressed individual's social network (e.g., spouse and relatives) (Wade \& Kendler, 2000). In this study, relationships were built over an 8-week period within an exercise group. Presumably, this period was not long enough for building deep and strong social ties, making it unlikely that participants perceived their teammates as intimates. Participants' ratings on the Group Integration-Social scale at the end of the intervention-the scores of which fell in a moderate range-seem to support this view.

In a similar vein, the length of time of this study may have minimized the effect of the group-based intervention. Our results revealed only that social relations developed within an exercise group over 8 weeks did not produce additional antidepressant effects, but this does not give any information about possible longterm effects of social relations. Implementing a team-building intervention over a longer period of time might have resulted in deeper relations among exercise group members, which in turn may have led them to consider their teammates as significant others, intimates. Again, future research is needed to explore the possible long-term effects of social relationships on the psychological benefits of exercise.

However, building strong social ties is not only a matter of time; it also requires meeting people with whom we feel attracted to. If small exercise classes are appropriate to develop task and social cohesion (Carron \& Spink, 1995), they do not provide sufficient opportunities to meet other attractive and interesting people (Carron, Hausenblas, \& Estabrooks, 2003). Therefore, the small size of the group that received a team-building intervention may also have contributed to the fact that no additional effect was found for the group-based intervention. Future research should consider larger groups in order to examine this point.

Thirdly, although data from the exercise group that received a team-building intervention did not result in greater alleviation in depressive symptoms for their members, it does not imply that all the individuals within the group did not benefit from the intervention. Indeed, Faulkner and Carless (2006) recently reemphasized the individual-specific nature of many mental disorders. Based on this fact, the authors argued that "because the effects of physical activity on mental health are likely to vary from person to person, it is possible that nomothetic analysis will mask important improvement made on an individual basis" (Faulkner \& Carless, 2006, p. 263). Consequently, the authors recommended that future research should adopt an appropriately broad theoretical stance and a full complement of suitably inclusive research methods in order to allow for the individual specificity to be considered.

Finally, it should be mentioned that the impact of combined treatments may be underestimated when the dependent measure restricts how low scores can be (which is known as a floor effect). Such an effect may apply to our study because (a) cohesion is a group property that takes time to develop (Loughead \& Carron, 2004; Loughead et al., 2001), but (b) BDI scores were substantially decreased in a short time (participants in the high-frequency exercise groups experienced a $35-40 \%$ reduction in their depressive symptoms by the fourth week of the study). 
Although our results make a good case for the value of frequent moderateto-vigorous aerobic exercise in a population of persons with moderate levels of clinical depression, this study has several noteworthy limitations. These mainly include small sample size and site-specific mode of recruitment. Small sample size is usually associated with an elevated risk of low statistical power and could explain the nonsignificant findings for our group-based intervention. Nevertheless, only trivial differences in BDI scores were noticed at both assessment times between participants in the HFex condition and those in the HFex + group-based intervention (Figure 1). Also, all participants in this study were recruited in a sport and fitness facility, which implies that they presumably had initial positive expectancies toward exercise and physical activity. Undoubtedly, this is detrimental to the external validity of our results. However, given the aims and constraints of this research (i.e., we wanted to have some persons with clinical depression exercise on a frequent basis for a duration of 8 weeks in a row), this sport and fitness facility enabled us (a) to reduce the effects of extraneous factors (e.g., weather conditions), and (b) to take advantage of comfortable rooms as well as efficient and easy-to-use equipment. Finally, the impact of our results may be undermined because (a) only those who completed the program were included in the analysis, (b) we supervised the program and evaluated the main outcome, and (c) we did not use any clinical diagnosis to classify our participants as "depressed" or "not depressed."

Nevertheless, some worthwhile findings resulted from this pilot study. To begin with, in contrast to well-established statements, individuals with elevated scores on objective measures of depression do not systematically benefit from physical activity of a moderate/vigorous nature. Indeed, frequent (three to five sessions per week) and infrequent (one session per week) aerobic forms of exercise performed at a moderate/vigorous relative intensity did not appear to be equally effective in treating depression. Second, social relations (operationalized as cohesion) within an exercise group did not produce short-term effects on depression. However, it cannot be excluded that longer team-building interventions result in deeper relations among teammates, which, in turn, might affect the relationship between physical activity and depression.

\section{Acknowledgments}

The authors are grateful to the management and staff of YNS for allowing free access to their facility throughout this study. They also thank the numerous undergraduate students from the Sport and Physical Education Faculty at the University of Reims who assisted in data collection.

\section{References}

American College of Sports Medicine (ACSM) (2000). Training guidelines for physical fitness and exercise performance. Baltimore, MD: Lippincott, Williams, \& Wilkins.

American Psychiatric Association (APA) (2000). Diagnostic and statistical manual of mental disorders (DSM-IV-TR) (4th ed.). Washington, DC: Author.

Andersson, L. (1998). Loneliness research and interventions: A review of the literature. Aging \& Mental Health, 2, 264-274.

Angst, J., \& Stassen, H.H. (2001). Do antidepressants really take several weeks to show effect? In B.E. Leonard (Ed.), Antidepressants (pp. 21-30). Boston: Birkhäuser Verlag. 
Bailey, M., \& McLaren, S. (2005). Physical activity alone or with others as predictors of sense of belonging and mental health in retirees. Aging \& Mental Health, 9, 82-90.

Beck, A.T., Steer, R.A., \& Brown, G.K. (1996). Inventaire de dépression de Beck-II [Beck-II depression inventory]. Paris: Editions du Centre de Psychologie Appliquée.

Berger, B.G., \& Motl, R.W. (2001). Physical activity and quality of life. In R.N. Singer, H.A. Hausenblas, \& C.M. Janelle (Eds.), Handbook of sport psychology (2nd ed.) (pp. 636-671). New York: John Wiley \& Sons.

Berger, B.G., \& Owen, D.R. (1988). Stress reduction and mood enhancement in four exercise modes: Swimming, body conditioning, hata yoga, and fencing. Research Quarterly for Exercise and Sport, 59, 148-159.

Blumenthal, J.A., Babyak, M.A., Moore, K.A., Craighead, W.E., Herman, S., Khatri, P., Waugh, R., Napolitano, M.A., Forman, L.M., Appelbaum, M., Doraiswamy, M., \& Krishnan, K.R. (1999). Effects of exercise training on older patients with major depression. Archives of Internal Medicine, 159, 2349-2356.

Booth, F.W., \& Lees, S.J. (2006). Physically active subjects should be the control group. Medicine \& Science in Sports \& Exercise, 38, 405-406.

Carron, A.V., \& Brawley, L.R. (2000). Cohesion: Conceptual and measurement issues. Small Group Research, 31, 89-106.

Carron, A.V., Brawley, L.R., \& Widmeyer, W.N. (1998). The measurement of cohesiveness in sport groups. In J.L. Duda (Ed.), Advances in sport and exercise psychology measurement (pp. 213-226). Morgantown, WV: Fitness Information Technology.

Carron, A.V., Hausenblas, H.A., \& Estabrooks, P.A. (2003). The psychology of physical activity. New York: McGraw-Hill.

Carron, A.V., \& Spink, K.S. (1993). Team building in an exercise setting. The Sport Psychologist, 7, 8-18.

Carron, A.V., \& Spink, K.S. (1995). The group size-cohesion relationship in minimal groups. Small Group Research, 26, 86-105.

Chaouloff, F. (1997). Effects of acute physical exercise on central serotoninergic systems. Medicine \& Science in Sports \& Exercise, 29, 58-62.

Coleman, D., \& Iso-Ahola, S.E. (1993). Leisure and health: The role of social support and self-determination. Journal of Leisure Research, 25, 111-128.

Cotman, C.W., \& Engesser-Cesar, C. (2002). Exercise enhances and protects brain function. Exercise \& Sport Sciences Review, 30(2), 75-79.

Courneya, K.S. (1995). Cohesion correlates with affect in structured exercise classes. Perceptual \& Motor Skills, 81, 1021-1022.

Craft, L.L. (2005). Exercise and clinical depression: Examining two psychological mechanisms. Psychology of Sport \& Exercise, 6, 151-171.

Craft, L.L. \& Landers, D.M. (1998). The effect of exercise on clinical depression and depression resulting from mental illness: A meta-analysis. Journal of Sport \& Exercise Psychology, 20, 339-357.

Deforche, B., \& De Bourdeaudhuij, I. (2000). Differences in psychosocial determinants of physical activity in older adults participating in organised versus non-organised activities. Journal of Sports Medicine \& Physical Fitness, 40, 362-362.

Dimeo, F., Bauer, M., Varahram, I., Proest, G., \& Halter, U. (2001). Benefits from aerobic exercise in patients with major depression: a pilot study. British Journal of Sports Medicine, 35, 114-117.

Dishman, R.K., Renner, K.J., White-Welkley, J.E., Burke, K.A., \& Bunnell, B.N. (2000). Treadmill exercise training augments brain norepinephrine response to familiar and novel stress. Brain Research Bulletin, 52, 337-342.

Dunn, A.L., Trivedi, M.H., Kampert, J.B., Clark, C.G., \& Chambliss, H.O. (2002). The DOSE study: A clinical trial to examine efficacy and dose response of exercise as treatment for depression. Control \& Clinical Trial, 23, 584-603. 
Dunn, A.L., Trivedi, M.H., Kampert, J.B., Clark, C.G., \& Chambliss, H.O. (2005). Exercise treatment for depression: Efficacy and dose-response. American Journal of Preventive Medicine, 28, 1-8.

Einarson, T.R., Iskedjian, M., Addis, A., \& Mittmann, N. (1998). Meta-analysis of venlafaxine in the treatment of major depressive disorder. Canadian Journal of Clinical Pharmacology, 5, 205-216.

Estabrooks, P.A., \& Carron, A.V. (1999). Group cohesion in older adult exercisers: Prediction and intervention effects. Journal of Behavioral Medicine, 22, 575-588.

Faulkner, G., \& Carless, D. (2006). Physical activity in the process of psychiatric rehabilitation: Theoretical and methodological issues. Psychiatric Rehabilitation Journal, 29, 258-266.

Fox, K.R. (1999). The influence of physical activity on mental well-being. Public Health Nutrition, 2, 411-418.

Greenberg, P.E., Kessler, R.C., Birnbaum, H.G., Leong, S.A., Lowe, S.W., Berglund, P.A., \& Corey-Lisle, P.K. (2003). The economic burden of depression in the United States: How did it change between 1990 and 2000? British Journal of Psychiatry, 183, 514-519.

Hansen, N.B., Lambert, M.J., \& Forman, E.M. (2002). The psychotherapy dose-response effect and its implications for treatment delivery services. Clinical Psychology: Science and Practice, 9, 329-343.

Henderson, A.S. (1998). Social support: Its present significance for psychiatric epidemiology. In B.P. Dohrenwend (Ed.), Adversity, stress, and psychopathology (pp. 390-397). New York: Oxford University Press.

Heuzé, J.P., \& Fontayne, P. (2002). Questionnaire sur l'Ambiance du Groupe: A Frenchlanguage instrument for measuring group cohesion. Journal of sport \& Exercise Psychology, 24, 42-67.

Hickson, R.C., Hagberg, J.M., Ehsani, A.A., \& Holloszy, J.O. (1981). Time course of the adaptive responses of aerobic power and heart rate to training. Medicine \& Science in Sport \& Exercise, 13, 17.

Hill, J.L., \& Estabrooks, P.A. (2000). The relationships between group interaction and cohesion in exercise classes for older adults. Paper presented at the meeting of the Association for the Advancement of Applied Sport Psychology. Nashville, TN.

Kessler, R.C., Berglund P., Demler, O., Jin, R., Koretz, D., Merikangas, K.R., Rush, A.J., Walters, E.E., \& Wang, P.S. (2003). The epidemiology of major depressive disorder: Results from the National Comorbidity Survey Replication (NCS-R). Journal of the American Medical Association, 289, 3095-3105.

Klein, M.H., Greist, J.H., Gurman, A.S., Neimeyer, R.A., Lesser, D.P., Bushnell, N.J., \& Smith, R.E. (1985). A comparative outcome study of group psychotherapy vs. exercise treatment for depression. International Journal of Mental Health, 13, 148-177.

Lawlor, D.A., \& Hopker, S.W. (2001). The effectiveness of exercise as an intervention in the management of depression: Systematic review and meta-regression analysis of randomised controlled trials. British Medical Journal, 322, 1-8.

Lepape, A. (1998). La mesure de la prévalence de la dépression dans les enquêtes du CREDES [Measurement of depression prevalence in CREDES surveys]. Paris: Centre de Recherches, d'Etudes et de Documentation en Economie de la Santé.

Loughead, T.M., \& Carron, A.V. (2004). The mediating role of cohesion in the leader behaviour-satisfaction relationship. Psychology of Sport and Exercise, 5, 355-371.

Loughead, T.M., Colman, M.M., \& Carron, A.V. (2001). Investigating the mediational relationship of leadership, class cohesion, and adherence in an exercise setting. Small Group Research, 32, 558-575.

Lyubomirski, S., \& Noelen-Hoeksema, S. (1995). Effects of self-focused rumination on negative thinking and interpersonal problem-solving. Journal of Personality \& Social Psychology, 69, 176-190. 
Martinsen, E.W., Hoffart, A., \& Solberg, O. (1989). Comparing aerobic and nonaerobic exercise in the treatment of clinical depression: A randomized trial. Comprehensive Psychiatry, 30, 324-331.

Martinsen, E.W., Medhus, A., \& Sandvik, L. (1985). Effects of aerobic exercise on depression: a controlled study. British Medical Journal, 291, 109.

Motl, R.W., Berger, B.G., \& Davis, S.L. (1997). High-intensity exercise and the acute mood states of trained cyclists. Journal of Sport \& Exercise Psychology, 19(Suppl.), S91.

Mutrie, N. (1988). Exercise as a treatment for moderate depression in the UK National Health Service (abstract). Proceedings of sport, health psychology, and exercise symposium (pp. 96-105). London: Sports Council and Health Education Authority.

Mutrie, N. (2000). The relationship between physical activity and clinically defined depession. In S.J.H. Biddle, K.R. Fow, \& S.H. Boutcher (Eds.), Physical activity and psychological well-being (pp. 46-62). London: Routledge.

North, T.C., McCullagh, P., \& Tran, Z.V. (1990). Effect of exercise on depression. Exercise \& Sport Sciences Reviews, 18, 379-415.

O'Neal, H.A., Dunn, A.L., \& Martinsen, E.W. (2000). Depression and exercise. International Journal of Sport \& Exercise Psychology, 31, 110-135.

Pate, R.R., Pratt, M., Blair, S.N., Haskell, W.L., Macera, C.A., Bouchard, C. et al. (1995). Physical activity and public health: a recommendation from the Centers for Disease Control and Prevention and the American College of Sports Medicine. Journal of the American Medical Association, 273(5), 402-407.

Petruzzello, S.J., \& Landers, D.M. (1994). Varying the duration of acute exercise: Implications for changes in affect. Anxiety, Stress, \& Coping, 6, 301-310.

Schulberg, H.C., Katon, W.J., Simon, G.E., \& Rush, A.J. (1999). Best clinical practice: Guidelines for managing major depression in primary medical care. Journal of Clinical Psychiatry, 60(7), 19-26.

Spitzer, R.L., Endicott, J., \& Robins, E. (1978). Research diagnostic criteria. Archives of General Psychiatry, 35, 773-782.

Stathi, A., Fox, K.R., \& McKenna, J. (2002). Physical activity and dimensions of subjective well-being in older adults. Journal of Aging \& Physical Activity, 10, 76-92.

Terry, P.C., Carron, A.V., Pink, M.J., Lane, A.M., Jones, G.J.W., \& Hall, M.P. (2000). Perceptions of group cohesion and mood in sport teams. Group Dynamics, 4, 244-253.

Thayer, R.E. (1996). The origin of everyday moods: Managing energy, tension, and stress. New York: Oxford Univeristy Press.

U.S. Department of Health \& Human Services (2000). Physical activity and fitness. In Healthy People 2010. Retrieved from www.health.gov/healthypeople/

Wade, T.D., \& Kendler, K.S. (2000). The relationship between social support and major depression: Cross-sectional, longitudinal, and genetic perspectives. Journal of Nervous \& Mental Diseases, 188, 251-258.

Widmeyer, W.N., \& Williams, J.M. (1991). Predicting cohesion in coacting sport. Small Group Research, 22, 548-570.

Zung, W.W.K. (1965). Self-rating depression scale. Archives of General Psychiatry, 12, 63-70.

Manuscript submitted: July 20, 2006

Revision accepted: January 15, 2007 